\title{
Wake up to insomnia: future approaches to the management of insomnia
}

\section{Mohammad Tahir Hussain Steven A. Shea}

Division of Sleep Medicine, Harvard Medical School, Brigham and Women's Hospital, Boston, MA, USA
Correspondence: Steven A. Shea Division of Sleep Medicine, Harvard Medical School, Brigham and Women's Hospital, 22I Longwood Avenue, Boston 02II5, MA, USA

Email sshea@hms.harvard.edu
This article was published in the following Dove Press journal:

Nature and Science of Sleep

25 January $201 \mathrm{I}$

Number of times this article has been viewed

Insomnia is rife. Acutely, almost every individual has experienced a poor night's sleep, and many short-term remedies for insomnia are tried, including over-the-counter sleep aids and alcohol. Chronic insomnia is the most common sleep disorder, affecting nearly 40 million Americans and accounting for nearly one-third of adults. The annual cost associated with this disorder in the US is estimated at over $\$ 15$ billion $^{1}$ and over $\$ 100$ billion for indirect costs, such as costs of self-medication, health care-related costs due to exacerbations of comorbid conditions, and costs of reduced productivity. ${ }^{2}$ Degradations of sleep quantity and sleep quality in insomnia have been associated with deleterious long-term effects, including cardiovascular, endocrine, psychological, and immune system complications. Despite these facts, chronic insomnia is not always perceived as a treatable and important medical condition. Symptoms are not always proffered by patients, and diagnoses can be ignored by physicians, partly because insomnia is so common that it is considered somewhat normal, particularly in elderly individuals. These days, recognized treatments for insomnia include pharmacological therapies and the more recently accepted behavioral therapies, including stimulus control, relaxation, and cognitive and behavioral therapy. Research to evaluate and formulate treatments for insomnia is often beset by the problem that insomnia is usually of multifactorial etiology among individuals. Thus, complex and important questions remain, eg, which types of insomnia can be cured, which therapy is best in each situation and in each patient, and why do so many untreated or suboptimally treated individuals with insomnia exist? There is also a clear need to examine the long-term effects of treatment on diverse cultures, ages, and races and in those with comorbid disorders, using randomized clinical trials of sufficient duration.

In the first full year of publication, the Nature and Science of Sleep (NSS) has published a set of articles that begins to examine the advantages and limitations of a number of relatively novel pharmacological and behavioral therapies for insomnia. These papers ought to help clinicians appraise new therapies, better manage insomnia, and identify important gaps in our knowledge and promising future approaches.

A number of papers in NSS review new pharmacological strategies for insomnia therapy, including the use of nonbenzodiazepine gamma-aminobutyric acid receptor agonists such as zolpidem, zaleplon, and eszopiclone (Z-drugs), which potentially have reduced adverse effects with improved safety and tolerance compared with older benzodiazepines; ${ }^{9}$ the melatonin receptor agonist ramelteon; ${ }^{12}$ serotonin receptor antagonists; ${ }^{6}$ and therapies with novel routes of administration, such as oral spray and 
sublingual tablets. ${ }^{7,11}$ For instance, administration of zolpidem via a rapid-absorption oral spray or a sublingual tablet can have advantages due to faster onset of action, lower dose requirement, better patient compliance and convenience, and increased bioavailability. Because the Z-drugs are rapid action, they could be used for short-term insomnia, with the precaution that fast-acting drugs could have potentially faster side effects and pose an increased risk for accidental overdose or abuse of the drugs. Moreover, further studies are needed to establish safety, particularly in terms of the carryover of excessive sleepiness into the daytime. ${ }^{11}$

Sleep patterns change with increasing age. Typically, sleep is lighter, more fragmented, and shorter, ${ }^{9}$ and the presence of comorbid conditions that interfere with sleep, such as chronic pain, depression, chronic obstructive pulmonary disease (COPD), and congestive heart failure, also lead to a higher prevalence of insomnia in the elderly. An article in NSS highlights the fact that insomnia is associated with an increased risk of hypertension and cardiovascular disease. ${ }^{13}$ This interrelationship between insomnia and disease can precipitate a vicious circle whereby insomnia can exacerbate disease, which can further exacerbate insomnia. Moreover, interpretation of clinical trials in the elderly becomes challenging due to confounding effects of medications for these comorbid conditions, and decreased clearance of sleep medications. To solve this challenging question, a paper in NSS specifically reviews pharmacotherapy for insomnia in the elderly ${ }^{4}$ and concludes that eszopiclone is well suited, improving quality of life without the development of drug tolerance and without rebound insomnia after discontinuation. Similarly, some promise was suggested for the use of 5-hydroxytryptamine $2 \mathrm{~A}\left(5-\mathrm{HT}_{2 \mathrm{~A}}\right)$ antagonists in treating sleep maintenance insomnia. ${ }^{6}$ Ramelteon was helpful in sleep onset insomnia and circadian rhythm disorder, with no rebound insomnia and reportedly no abuse potential. Ramelteon also did not exacerbate respiratory disorders such as sleep apnea and COPD, which is a concern for other sedative medications that can depress ventilation or arousal responses. ${ }^{12}$ Understanding the molecular and receptor mechanisms involved in promoting sleep in varied disorders could provide future approaches in new drug development. In the long term, more randomized controlled trials are needed to assess both short-term and long-term effects of these medications and their efficacy in comorbid diseases that affect sleep quality or quantity.

In search of a better therapeutic approach for insomnia there is an emerging debate about using pharmacotherapy and behavioral therapy to manage insomnia. One of the first studies to formally address this debate using a randomized placebo-controlled trial was published in 1999 and demonstrated that a combined pharmacological and cognitive behavioral therapy for insomnia (CBT-I) was very effective, particularly in terms of duration of effect. Although this was the largest clinical trial in the field at that time, the sample size was relatively small $(\mathrm{n}=78)$, included only elderly people, and the pharmacotherapy included one medication, temazepam. Thus, this result cannot be generalized to a wider population or other drugs. ${ }^{3}$

In this regard, recent papers in NSS have begun to examine CBT-I in varied populations. For instance, a pilot randomized controlled crossover study in breast cancer survivor patients suggested that individualized CBT-I therapy sessions continued to improve sleep quality. ${ }^{10}$ Furthermore, two studies examined the efficacy of CBT-I in pediatric populations and showed promising results. ${ }^{14,15}$ Novel multimodal controlled therapies in children aged 5-10 years combined with training sessions with their parents revealed better efficacy and improved compliance. ${ }^{14}$ This novel ageappropriate treatment program was also very effective in improving sleep efficiency in children aged 11-17 years, possibly reflecting better acceptance in this age group. ${ }^{15}$

CBT-I contains components such as psychotherapy, sleep restriction, paradoxical intention, stimulus control, sleep hygiene, relaxation training, and biofeedback. These techniques can be effective in many patients, but a common problem is accessibility to CBT-I. ${ }^{5}$ Also, the relative efficacy of individual psychological therapeutic techniques within the components of CBT-I still needs to be evaluated in appropriately controlled studies.

Additional individual novel therapies for insomnia are reviewed in NSS, such as Internet-based interventions, bright light treatments, intensive sleep retraining, mindfulness meditation, and acupuncture, and these are assessed for comparative effectiveness with the goal of improved outcomes, reduced costs, and/or improved compliance. Many of these approaches have encouraging findings but still require further research, including randomized placebocontrolled trials, to establish their effectiveness. ${ }^{8}$

Finally, due to the importance of adequate insomnia management, in its first full year, NSS has published numerous original articles that highlight the relative efficacy of specific therapies over others and in specific patient populations. Specifically, NSS has published 12 articles on novel pharmacological and cognitive behavioral therapeutic methods for relieving insomnia in varied populations, including children, adolescents, adults, and the elderly. Additionally, NSS has published focused reviews on insomnia with comorbid 
conditions, as well as other novel therapeutic options. These informative reviews point out that there is a need for future long-term clinical trials. As mentioned previously, due to the complexity and multifactorial etiology of insomnia, many questions remain unanswered. We encourage you to continue your research to provide tailored approaches to managing insomnia in specific populations or individuals, to publish your research in NSS, and to help put insomnia to sleep.

\section{References}

1. Rosekind MR, Gregory KB. Insomnia risks and costs: health, safety, and quality of life. Am J Manag Care. 2010;16(8);617-626.

2. Matteson-Rusby SE, Pigeon WR, Gehrman P, Perlis ML. Why treat insomnia? Prim Care Companion J Clin Psychiatry. 2010;12(1): PCC.08r00743.

3. Morin CM, Colecchi C, Stone J, et al. Behavioral and pharmacological therapies for late-life insomnia: a randomized controlled trial. JAMA. 1999;281(11):991-999.

4. Kirkwood C, Breden E. Management of insomnia in elderly patients using eszopiclone. Nat Sci Sleep. 2010;2:151-158.

5. Unbehaun T, Spiegelhalder K, Hirscher V, Riemann D. Management of insomnia: update and new approaches. Nat Sci Sleep. 2010;2:127-138.
6. Vanover KE, Davis RE. Role of 5-HT ${ }_{24}$ receptor antagonists in the treatment of insomnia. Nat Sci Sleep. 2010;2:139-150.

7. Ownby RL. New approaches in the treatment of short term and middle of the night insomnia: emerging evidence for the role of sublingual zolpidem tablets. Nat Sci Sleep. 2010;2:63-69.

8. Dautovich ND, McNamara J, Cross NJ, McCrae CS. Tackling sleeplessness: psychological treatment options for insomnia. Nat Sci Sleep. 2010;2:23-37.

9. Dzierzewski JM, O’Brien EM, Kay D, McCrae CS. Tackling sleeplessness: psychological treatment options for insomnia in older adults. Nat Sci Sleep. 2010;2:47-61.

10. Fiorentino L, McQuaid JR, Liu L, et al. Individual cognitive behavioral therapy for insomnia in breast cancer survivors: a randomized controlled crossover pilot study. Nat Sci Sleep. 2010;2:1-8.

11. Neubauer DN. Zolpimist: a new formulation of zolpidem tartrate for the short-term treatment of insomnia in the US. Nat Sci Sleep. 2010; 2:79-84.

12. Mets MAJ, Daventer KR, Olivier B, Verster JC. Critical appraisal of ramelteon in the treatment of insomnia. Nat Sci Sleep. 2010;2: 257-266.

13. Spiegelhalder K, Scholtes C, Riemann D. The association between insomnia and cardiovascular diseases. Nat Sci Sleep. 2010;2:71-78.

14. Schlarb AA, Schurian KV, Poets CF, Hautzinger M. First effects of a multicomponent treatment for sleep disorders in children. Nat Sci Sleep. 2011;3:1-11.

15. Schlarb AA, Liddle CC, Hautzinger M. JuSt - a multimodal program for treatment of insomnia in adolescents: a pilot study. Nat Sci Sleep. 2011;3:13-20.

\section{Publish your work in this journal}

Nature and Science of Sleep is an international, peer-reviewed, open access journal covering all aspects of sleep science and sleep medicine, including the neurophysiology and functions of sleep, the genetics of sleep, sleep and society, biological rhythms, dreaming, sleep disorders and therapy, and strategies to optimize healthy sleep. The journal welcomes

\section{Dovepress}

original research, clinical \& epidemiological studies, reviews \& evaluations, case reports and extended reports. The manuscript management system is completely online and includes a very quick and fair peerreview system, which is all easy to use. Visit http://www.dovepress.com/ testimonials.php to read real quotes from published authors. 